\title{
POINCARÉ SERIES OF MODULES OVER LOCAL RINGS
}

\author{
GERSON LEVIN
}

\begin{abstract}
There is a well known conjecture, originally made by Kaplansky, that the Poincare series $P_{R}^{M}(z)$ of a finitely generated module $M$ over a local ring $R$ is a rational function. Two reductions of this conjecture are made, one to the case where $M$ is artinian (for a fixed $R$ ) and the other to the case where $R$ is artinian.
\end{abstract}

Introduction. Let $R$ be a local ring with maximal ideal $\mathbf{m}$ and residue field $k=R / \mathbf{m}$. All $R$-modules considered are assumed to be finitely generated and all unspecified tensor products are over $R$.

For any $R$-module $M$, we may consider the formal power series

$$
P_{R}^{M}(z)=\sum_{i=0}^{\infty} B_{i} z^{i}
$$

where $B_{i}=\operatorname{dim}_{k} \operatorname{Tor}_{i}^{R}(M, k)$. There is a well-known conjecture, originally made by Kaplansky, that $P_{R}^{M}(z)$ is a rational function for all local rings $R$ and all $R$-modules $M$. We make two reductions of this conjecture.

THEOREM 1. The series $P_{R}^{M}$ is rational for all local rings $R$ and $R$-modules $M$ if and only if $P_{R}^{M}$ is rational for all artinian local rings $R$ and $R$-modules $M$.

THEOREM 2. For any local ring $R, P_{R}^{M}$ is rational for all $R$-modules $M$ if and only if $P_{R}^{M}$ is rational for all artinian $R$-modules $M$.

The proofs will follow after some lemmas. Theorem 1 for the case $M=k$ first appeared in [3]. However, the proof included here is much shorter. In [2], Gulliksen and Ghione note that the author's result together with a result of Gulliksen's imply that $P_{R}^{M}$ is rational for all local rings $R$ and $R$-modules $M$ if and only if $P_{R}^{k}$ is rational for all artinian local rings $R$. Theorem 2 is new.

Lemma 1. Let $K$ be the Koszul complex of $R$ and $M$ an $R$-module. Then there is an integer $n_{0}$ such that for all $n \geqslant n_{0}$, the induced homomorphism

$$
H\left(\mathbf{m}^{n+1} M \otimes K\right) \rightarrow H\left(\mathbf{m}^{n} M \otimes K\right)
$$

is zero.

Proof. Since

$$
H\left(\mathbf{m}^{n} M \otimes K\right)=\frac{\left(\mathbf{m}^{n} M \otimes K\right) \cap Z(M \otimes K)}{\mathbf{m}^{n} B(M \otimes K)}
$$

Received by the editors December 20, 1976.

AMS (MOS) subject classifications (1970). Primary 13D99. 
and since $H\left(\mathrm{~m}^{n} M \otimes K\right)$ is a vector space over $k$,

$$
\mathbf{m}\left(\left(\mathbf{m}^{n} M \otimes K\right) \cap Z(M \otimes K)\right) \subset \mathbf{m}^{n} B(M \otimes K) .
$$

Then by the Artin-Rees lemma, there is an integer $n_{0}$ such that for $n \geqslant n_{0}$,

$$
\begin{aligned}
\mathbf{m}^{n+1}(M \otimes K) \cap Z(M \otimes K) & \subset \mathbf{m}\left(\mathbf{m}^{n}(M \otimes K) \cap Z(M \otimes K)\right) \\
& \subset \mathbf{m}^{n} B(M \otimes K) .
\end{aligned}
$$

Thus the induced homomorphism

$$
H\left(\mathbf{m}^{n+1} M \otimes K\right) \rightarrow H\left(\mathbf{m}^{n} M \otimes K\right)
$$

is zero.

LeMma 2. Let $A$ be a differential graded $R$-algebra and $E$ a differential graded $A$-module which is free as a graded A-module (i.e. forgetting differentials) and such that $d E \subset \mathbf{m} E$. Let $M$ and $N$ be $R$-modules such that $\mathbf{m} M \subset N \subset M$, and such that the canonical map $g: N \otimes E \rightarrow M \otimes E$ is injective. Then if the induced homomorphism $H(N \otimes A) \rightarrow H(M \otimes A)$ is zero, so is the induced homomorphism $H(N \otimes E) \rightarrow H(M \otimes E)$.

Proof. Assume that $H(N \otimes A) \rightarrow H(M \otimes A)$ is zero.

Then

$$
f(Z(N \otimes A)) \subset B(M \otimes A),
$$

where $f$ is the canonical map $f: N \otimes A \rightarrow M \otimes A$. Define a filtration on $E$ as follows:

$$
F_{p} E=\sum_{i>p} A_{i} E
$$

We first show that

$$
g\left(Z(N \otimes E) \cap\left(N \otimes F_{p} E\right)\right) \subset B_{p}(M \otimes A) E+g\left(N \otimes F_{p+1} E\right) .
$$

Let $S_{1}, S_{2}, \ldots$ be the elements of degree $n-p$ of a homogeneous $A$-basis for $E$. Then if $z \in Z(N \otimes E) \cap\left(N \otimes F_{p} E\right)$, there are $a_{i} \in N \otimes A_{p}$ such that $z-\sum a_{i} S_{i} \in N \otimes F_{p+1} E$. It follows that $\Sigma\left(d a_{i}\right) S_{i} \in N \otimes F_{p} E$ so each $d a_{i}=0$. But then because of (1), each $f\left(a_{i}\right) \in B_{p}(M \otimes A)$ and since $g\left(\sum a_{i} S_{i}\right)$ $=\sum f\left(a_{i}\right) S_{i}$, (2) is proved.

Next, observe that the derivation formula

$$
d(x y)=(d x) y+(-1)^{\operatorname{deg} x} x(d y)
$$

shows that

$$
\left(d\left(M \otimes A_{p+1}\right)\right) E \subset d\left(\left(M \otimes A_{p+1}\right) E\right)+\left(M \otimes A_{p+1}\right) d E
$$

and since $d E \subset \mathbf{m} E$ and $\mathbf{m} M \subset N$,

$$
\left(d\left(M \otimes A_{p+1}\right)\right) E \subset d(M \otimes E)+g\left(N \otimes F_{p+1} E\right) .
$$

Now by (2) and (3) and the injectivity of $g$, 


$$
\begin{aligned}
g(Z(N \otimes E) & \left.\cap\left(N \otimes F_{p} E\right)\right) \\
& \subset g\left(Z(N \otimes E) \cap\left(N \otimes F_{p+1} E\right)\right)+B(M \otimes E) .
\end{aligned}
$$

Since $E$ is $A$-free, $F_{0} E=E$ and also for any $n, F_{p} E \cap E_{n}=0$ for $p>n$. Hence $g(Z(N \otimes E)) \subset B(M \otimes E)$ and $H(N \otimes E) \rightarrow H(M \otimes E)$ is zero.

Proof of TheOREM 2. By Lemma 1 , there exists an integer $n_{0}$ such that for $n \geqslant n_{0}$

$$
H\left(\mathbf{m}^{n+1} M \otimes K\right) \rightarrow H\left(\mathbf{m}^{n} M \otimes K\right)
$$

is zero. Let $X$ be a minimal free resolution of $k$. By [1], $X$ is free as a graded $K$-module. Thus Lemma 2 applies showing that

$$
H\left(\mathbf{m}^{n+1} M \otimes X\right) \rightarrow H\left(\mathbf{m}^{n} M \otimes X\right)
$$

is zero for $n \geqslant n_{0}$. It also follows that

$$
H\left(\mathbf{m}^{n+1} M \otimes X\right) \rightarrow H(M \otimes X)
$$

is zero for $n \geqslant n_{0}$.

Thus the short exact sequences

$$
0 \rightarrow \mathbf{m}^{n} M \rightarrow M \rightarrow M / \mathbf{m}^{n} M \rightarrow 0
$$

induce short exact sequences

$$
0 \rightarrow \operatorname{Tor}_{p}^{R}(M, k) \rightarrow \operatorname{Tor}_{p}^{R}\left(M / \mathbf{m}^{n} M, k\right) \rightarrow \operatorname{Tor}_{p-1}^{R}\left(\mathbf{m}^{n} M, k\right) \rightarrow 0
$$

for $n \geqslant n_{0}+1$, showing that

$$
P_{R}^{M / \mathrm{m}^{n} M}=P_{R}^{M}+z P_{R}^{m^{n} M} .
$$

We now compute $P_{R}^{m^{n} M}$ for $n \geqslant n_{0}+1$. Because of (4), the short exact sequences

$$
0 \rightarrow \mathbf{m}^{n+i+1} M \rightarrow \mathbf{m}^{n+i} M \rightarrow \mathbf{m}^{n+i} M / \mathbf{m}^{n+i+1} M \rightarrow 0
$$

induce short exact sequences

$$
\begin{aligned}
0 & \rightarrow \operatorname{Tor}_{p}^{R}\left(\mathbf{m}^{n+i} M, k\right) \rightarrow \operatorname{Tor}_{p}^{R}(k, k) \otimes\left(\mathbf{m}^{n+i} M / \mathbf{m}^{n+i+1} M\right) \\
& \rightarrow \operatorname{Tor}_{p-1}^{R}\left(\mathbf{m}^{n+i+1} M, k\right) \rightarrow 0
\end{aligned}
$$

and thus

$$
c_{n+i} P_{R}^{k}=P_{R}^{\mathrm{m}^{n+i} M}+z P_{R}^{\mathrm{m}^{n+i+1} M}
$$

for all $i \geqslant 0$ where

$$
c_{r}=\operatorname{dim}_{k} \mathbf{m}^{r} M / \mathbf{m}^{r+1} M .
$$

Let $H(z)=\sum_{i=0}^{\infty} c_{n+i} z^{i}$. By the Hilbert theory, $c_{n+i}$ is a polynomial in $i$ for large $i$ so $H(z)$ is a rational function. Now

$$
P_{R}^{\mathbf{m}^{n} M}=\sum_{i=0}^{\infty}(-1)^{i} z^{i} c_{n+i} P_{R}^{k}=H(-z) P_{R}^{k}
$$

Then (5) and (6) show that for $n \geqslant n_{0}+1, P_{R}^{M}$ is rational if both $P_{R}^{M / \mathrm{m}^{n} M}$ and $P_{R}^{k}$ are rational. Since $M / \mathbf{m}^{n} M$ and $k$ are artinian modules the theorem follows. 
Proof of Theorem 1. Assume that all modules over artinian local rings have rational Poincare series and let $R$ be any local ring and $M$ an $R$-module. By Theorem 2, it is enough to prove that $P_{R}^{M}$ is rational for artinian $R$-modules $M$. So assume that $\mathrm{m}^{r} M=0$.

As in the proof of Theorem 1, there is an integer $n_{0}$ such that for $n \geqslant n_{0}$

$$
\operatorname{Tor}^{R}\left(\mathbf{m}^{n+1}, k\right) \rightarrow \operatorname{Tor}^{R}\left(\mathbf{m}^{n}, k\right)
$$

is zero. Let $n>\max \left(r, n_{0}\right)$.

Then from (6)

$$
P_{R}^{\mathrm{m}^{n+1}}=H(-z) P_{R}^{k}
$$

where $H(z)=\sum_{i=0}^{\infty} \operatorname{dim}\left(\mathbf{m}^{n+i+1} / \mathbf{m}^{n+i+2}\right) z^{i}$. So

$$
P_{R}^{R / \mathbf{m}^{n+1}}=1+z H(-z) P_{R}^{k}
$$

We will show that for any $R$-module $N$ such that $\mathbf{m}^{n} N=0$,

$$
P_{R / \mathrm{m}^{n+1}}^{N}=\frac{P_{R}^{N}}{1-z\left(P_{R}^{R / \mathrm{m}^{n+1}}-1\right)} .
$$

With $N=k$, this shows that $P_{R}^{k}$ is rational and thus that $P_{R}^{R / \mathbf{m}^{n+1}}$ is rational. Then with $N=M$, it follows that $P_{R}^{M}$ is rational.

To prove (8), let $X$ be a minimal algebra resolution [1] of $k$ over $R$. We put $R^{*}=R / \mathrm{m}^{n+1}, m^{*}=\mathrm{m} / \mathrm{m}^{n+1}$, and $X^{*}=X \otimes R^{*}$. For each $i>2$, let $F_{i}$ be a free $R^{*}$-module such that

$$
F_{i} \otimes k \cong H_{i-1}\left(X^{*}\right)
$$

Because of (7) $H\left(\mathrm{~m}^{n+1} X\right) \rightarrow H\left(\mathrm{~m}^{n} X\right)$ is zero so $H\left(\mathrm{~m}^{n} X^{*}\right) \rightarrow H\left(X^{*}\right)$ is surjective. Thus we can choose $R$-homomorphism $\eta: F_{i} \rightarrow \mathbf{m}^{n} X_{i-1}^{*}$ such that the diagrams

$$
\begin{array}{ccc}
F_{i} & \rightarrow & \mathbf{m}^{n} X_{i-1}^{*} \\
\downarrow & & \downarrow \\
F_{i} \otimes k & \approx & H_{i-1}\left(X^{*}\right)
\end{array}
$$

commute. Now define a complex $Y=X^{*} \otimes T(F)$ with differential given by

$$
\begin{aligned}
d\left(x \otimes f_{1} \otimes \cdots \otimes f_{r}\right)= & d x \otimes f_{1} \otimes \cdots \otimes f_{r} \\
& +(-1)^{\operatorname{deg} x} x \eta\left(f_{1}\right) \otimes f_{2} \otimes \cdots \otimes f_{r}
\end{aligned}
$$

for $x \in X^{*}$ and $f_{i} \in F$.

It is easily seen that $d^{2}=0$ and that

$$
d(y \otimes f)=d y \otimes f\left(\bmod X^{*}\right)
$$

for $y \in Y$. Clearly $d Y \subset \mathbf{m}^{*} Y$. The definition of $\eta$ shows that

$$
Z_{i}\left(X^{*}\right) \subset B_{i}\left(X^{*} \oplus F\right) \subset B_{i}(Y)
$$

for $i>0$. If $x+f \in Z_{i}\left(X^{*} \oplus F\right)$ then $\eta(f) \in B_{i-1}\left(X^{*}\right)$ so

$$
f \in \mathbf{m}^{*} F_{i} \subset d\left(X_{1}^{*} \otimes F_{i}\right)+X_{i}^{*} .
$$


Thus

$$
Z_{i}\left(X^{*} \oplus F\right) \subset B_{i}\left(X^{*} \oplus F \oplus\left(X^{*} \otimes F\right)\right) .
$$

In particular, $H_{1}(Y)=H_{2}(Y)=0$. Assume that $H_{i}(Y)=0$ for $i<p$. By (9)

$Z_{p}(Y) \subset X^{*} \oplus F \oplus \coprod_{i=1}^{p-2}\left(Z_{i}(Y) \otimes F_{p-i}\right) \subset X^{*} \oplus F \oplus \coprod_{i=1}^{p-2} d\left(Y_{i+1} \otimes F_{p-i}\right)$

by induction. So by (10) $Y$ is acyclic and hence a minimal resolution of $k$ over $R^{*}$. Since $\mathbf{m}^{n} N=0$, the differential in $N \otimes Y$ is given by $d\left(x \otimes f_{1}\right.$ $\left.\otimes \cdots \otimes f_{n}\right)=d x \otimes f_{1} \otimes \cdots \otimes f_{n}$ for $x \in N \otimes X^{*}, f_{i} \in F$. Thus

$$
H(N \otimes Y) \cong H\left(N \otimes X^{*}\right) \otimes T(F) \cong H(N \otimes X) \otimes T(F) .
$$

Formula (8) follows.

COROllary. Let $R$ be a local ring and $M$ an $R$-module of finite length. Then there is an integer $n_{0}$ such that for $n \geqslant n_{0}$,

$$
P_{R / \mathrm{m}^{n}}^{M}=P_{R}^{M} /\left(1-z^{2} H(-z) P_{R}^{k}\right)
$$

where

$$
H(z)=\sum_{i=0}^{\infty} \operatorname{dim}_{k}\left(\mathbf{m}^{n+i} / \mathbf{m}^{n+i+1}\right) z^{i}
$$

\section{REFERENCES}

1. T. H. Gulliksen, $A$ proof of the existence of minimal $R$-algebra resolutions, Acta Math. 120 (1968), 53-58.

2. F. Ghione and T. H. Gulliksen, Some reduction formulas for the Poincaré series of modules, Univ. of Oslo (preprint).

3. G. Levin, Local rings and Golod homomorphisms, J. Algebra 37 (1975), 266-289.

Department of Mathematics, Brooklyn College (CUNY), Brooklyn, New York 11210 